\title{
Jenseits von Hume Demea. Eine Rehabilitierung in systematischer Absicht
}

\section{Journal Article}

\section{Author(s):}

von Sass, Hartmut

Publication date:

2010-12

Permanent link:

https://doi.org/10.3929/ethz-b-000029559

\section{Rights / license:}

In Copyright - Non-Commercial Use Permitted

\section{Originally published in:}

Neue Zeitschrift für systematische Theologie und Religionsphilosophie 52(4), https://doi.org/10.1515/NZST.2010.024 


\title{
Jenseits von Hume: Demea. Eine Rehabilitierung in systematischer Absicht
}

\author{
Hartmut von Sass \\ Theologische Fakultät der Universität Zürich, Kirchgasse 9, CH 8001 Zürich, Schweiz, \\ hartmutvonsass@access.uzh.ch
}

für Martin Möller

\section{Zum Auftakt}

Es fällt nicht immer leicht, zu bemerken, wann es Zeit ist, zu gehen. Verunglückte Abgänge sind daher so legendär wie zahlreich, sodass man für gelungene Abschiede schon dankbar ist. Auch die berühmten Dialogues concerning Natural Religion von David Hume (1711-1776) enthalten eine solche Wendung. Dort heißt es am Ende des vorletzten Teils von einem der Gesprächspartner:

Demea did not at all relish the latter part of the discourse; and he took occasion soon after, on some pretence or other, to leave the company. ${ }^{1}$

Demeas vorzeitiges Verschwinden hat Humes Kommentatoren bislang kaum beschäftigt, wie überhaupt die beiden anderen >Dia-Logiker`, Philo und Cleanthes, die Aufmerksamkeit ganz für sich beanspruchten. Dies hat durchaus gute Gründe, achtet man darauf, wie die Redeanteile, das argumentative Engagement und die intellektuelle Wendigkeit auf die drei Figuren verteilt sind: Die Dialogues werden zweifelsohne von Philo und Cleanthes dominiert, während Demeas Abgang wenn nicht als 'gelungen`, so doch immerhin als gerade noch rechtzeitig vollzogene Schadensbegrenzung durchgehen könnte - besonders wenn man sich die gedanklichen Sackgassen verdeutlicht, in die er sich zusehends hinein manövriert hat; so jedenfalls das traditionelle Bild.

An diesem Bild hat sich, wie wir sehen werden, einiges, kaum aber Entscheidendes gewandelt; denn das neue - nicht: erneute - Interesse an Demea verweigert ihm weiterhin eine echte Wertschätzung. Die entflammte philosophische und literaturwissenschaftliche Aufmerksamkeit für seine Stellung, vor allem aber für seine dialogische Funktion gleicht dadurch einem lediglich präziser vorgetragenen Angriff auf seine Sicht. Dies ist nicht verwunderlich, setzt man die von Hume selbst vorgelegte Charakterisierung

1 David Hume, Dialogues concerning Natural Religion. And Other Writings, hg. v. Dorothy Coleman, Cambridge 2007, 88; die Seitenzahlen in Klammern beziehen sich auf diesen Text. 
der drei Figuren an, die der Autor dem `neutralen $>$ Pamphilus - also >dem, der alle gern hat - in den Mund legt. Dieser hat seinen Auftritt in der Rahmenhandlung der Dialoge und berichtet einem Freund - und damit dem Leser - von der Debatte, die er zuvor gespannt verfolgte. Pamphilus also stellt den nun nicht unbedingt neutralen Steckbrief vor, welcher in Philo einen >careless sceptic erkennt, in Cleanthes den »accurate philosophical turn « des Theismus verwirklicht sieht, um Demea als Vertreter einer »rigide inflexible orthodoxy « vorzustellen (5). ${ }^{2}$ Das aufkommende Interesse an Letzterem stellt diese Charakteristik samt der entsprechenden Beurteilung kaum in Frage. Eher umgekehrt manifestiert sich dieses Interesse gerade darin, dass Demeas Scheitern nicht allein als argumentative Inszenierung, vielmehr als dialogisch eingefädelte Ausweglosigkeit jeder Orthodoxie angesehen wird. ${ }^{3}$

Wenn für den folgenden Gedankengang eine >Rehabilitierung des schwächsten Glieds in Humes Dialogen angekündigt wird, ist dies eine Reaktion auf ein doppeltes Unbehagen: Einerseits halte ich das straditionelle Übergehen Demeas und dessen selten direkt einsehbarem Anliegen für verdächtig und unbefriedigend, weshalb ich die sneue Hinwendung zu diesem Außenseiter begrüße. Andererseits verbirgt sich in dieser Hinwendung, so mein Eindruck, nur ein sneues` Mittel des eigentlich alten Unternehmens, welches Demea schlicht den Garaus machen will. Und so teile ich zwar das 'neue Interesse an ihm, ohne ihm die straditionell a ausbleibende Wertschätzung vorzuenthalten. Oder unmittelbarer und thetisch formuliert: In Demea begegnen wir einem möglichen Zugang zum religiösen Glauben, der Treffenderes von seinem Gegenstand zumindest anzudeuten weiß als Philos destruktive Kritik oder Cleanthes' naiver Theismus. Dadurch wird auch Demeas vorzeitiger Abschied vom sargumentativen Trauerspiek seiner beiden Kontrahenten in etwas anderem Licht erscheinen. So mag nun deutlicher sein, inwiefern es um eine >Rehabilitierung in systematischer Absicht gehen könnte.

Die Wendung ssystematisch steht hier in Abgrenzung von einem rein exegetischen Anliegen; denn eines der immer wieder bemühten Argumente dafür, sich auf den Disput zwischen Philo und Cleanthes zu beschränken, beruft sich auf die Ansicht, Philo tendenziell mit Humes eigener Position zu identifizieren, und Cleanthes' Sicht als deren zeitgenössisch profundeste Al-

2 Nach der Einschätzung Thomas Olshewskys fällt es schwerer, Demea ein treffendes Etikett zu geben im Vergleich zum skeptisch-religionskritischen Denken Philos und dem affirmativ-theistischen Ansatz von Cleanthes (so Thomas M. Olshewsky, Demea's Dilemmas, in: BSHP 11:3 (2003), 473-492, hier: 475).

3 Wiederum Pamphilus weist auf die Vorzüge hin, die die Gattung des Dialogs für Themen mit sich bringt, die zwar robvious and important, zugleich aber robscure and uncertain sind (vgl. 4); und es überrascht nun kaum, dass diese Skizze auf Humes eigentliches Thema, die Aussichten einer natürlichen Religion, zutrifft, ist diese Kurzbeschreibung doch bereits von diesem Anliegen her formuliert. - Nach Michael Prince markiert Humes Werk den Höhepunkt, aber auch "the end of religious dialogue" (Michael B. Prince, Hume and the End of Religious Dialogue, in: ASECS 25:3 (1992), 283-308, hier: 308). 
ternative anzusetzen. ${ }^{4}$ Demea hingegen zu vernachlässigen erscheint dann als angebracht, zumal Hume dessen Auftritte gewieft-ironisch auf's fast Lächerliche reduziert. In diesem Fall wäre seine Orthodoxie nicht mit >Rechtgläubigkeit`zu übersetzen, sondern mit `Dogmatismus`als einer Unverbesserlichkeit, die jede ernsthafte Beschäftigung mit ihr eigenhändig untergräbt.

Ich stimme diesen drei (exegetischen) Momenten - Humes Nähe zu Philo, seiner Distanz zu Demea und dem Respekt gegenüber Cleanthes - durchaus zu, sodass eine >Rehabilitierung in systematischer Absicht $<$ keinesfalls behaupten wird, der intentio auctoris zu entsprechen. Weit eher ist sie contra Hume verfasst, und zwar in bestimmtem Sinn: In seiner Skepsis gegenüber den Aussichten religiösen Glaubens ist Hume nicht skeptisch genug gewesen, ${ }^{5}$ weswegen er es bei Philos Widerlegung von Cleanthes' Verteidigungen belassen hat und nicht erkannte, dass der abseits stehende Demea die Bivalenz von Religionskritik auf der einen Seite und den theistischen metaphysica auf der anderen unterläuft. Auf welchem Wege nun gerade »the poor old Demea ${ }^{6}$ an einen Ort >jenseits von Hume ${ }^{6}$ führen könnte und dadurch nicht im 18. Jahrhundert verbleiben muss, sondern ungemein aktuell ist - um nicht den theologisch überreizten Term sanschlussfähig< zu verwenden -, möchte ich nun darlegen.

Dies wird in drei Schritten geschehen. Zunächst ist an Cleanthes' Analogieargument als Kern seiner Verteidigung berechtigten Glaubens zu erinnern, was uns zu einem Überblick der entsprechenden Einwände Philos, vor allem aber zur Kritik auch noch dieser Kritik führen wird (II.). Es schließt sich eine knappe Beschreibung dessen an, was Philo nach seinen Einsprüchen als Minimalversion religiösen Glaubens zu akzeptieren bereit ist, wobei sich dieses Zugeständnis im Ambivalenten zwischen Konfusion oder Raffinesse bewegt (III.). Dadurch ist eben diejenige Konstellation vorbereitet, die uns erlaubt, Demea als jemanden zu verstehen, den sein eigener `Erzeuger nicht zu erkennen vermochte: einen etwas wortkargen Vertreter einer auch heute ernst zu nehmenden, vielleicht sogar sympathischen Orthodoxie (IV.)!

\section{Cleanthes' Analogie und Philos Einspruch}

Es ist kaum damit zu rechnen, dass bei Hume, einem Meister der Anspielung, die Namensvergabe in seinen Dialogen von philosophischen Erwägungen unberührt bliebe. Und so ist die Existenz eines historischen Cle-

\footnotetext{
4 So etwa Norbert Hoerster, "Nachwort« zur deutschen Ausgabe der Dialoge, Stuttgart 2004, 147-158, hier: 151.

5 Die Vermutung, der Skeptiker Hume sei snicht skeptisch genug gewesen, findet sich bereits (wenn auch in anderer Akzentuierung) bei Norman KeMP SMITH, "Introduction " zu den Dialogues, Indianapolis/New York o.J., 11.

6 Olshewsky (s.o. Anm. 2), 473.
} 
anthes sehr gut belegt; es handelt sich wohl um einen etwa 300 bis 230 vor Christus lebenden Anhänger des Stoikers Zenon, der in seinem Hymnus auf Zeus das Universum als ein lebendiges Sein und Gott als dessen Seele besungen hat. Spurenelemente dieser Sicht finden wir auch in Humes Kreatur, die durch das klassisch vorgetragene Analogieargument für die Existenz - und Natur - Gottes weite Teile der Dialoge thematisch bestimmt - eine ganz kurze Erinnerung:

\section{II.1. Cleanthes' Analogie}

Die Teile II bis VIII der Dialoge enthalten die kritische Sichtung eines Arguments, dessen Erfolg Cleanthes als notwendige Bedingung dafür ansieht, rechtmäßig an Gott glauben zu können (vgl. 20). Ein argumentatives Scheitern müsste demnach in den Religionsverzicht münden, womit sich der Theist Cleanthes auf gleich zwei Thesen verpflichtet: Zum einen sei sein Argument wasserdicht, denn er selbst glaubt an Gott; zum anderen sei sein Argument das einzig angemessene, denn er lehnt alle alternativen Erwägungen ab, um sich ganz der Verteidigung seiner Auffassung zu verschreiben. An Demea gewandt lädt er schließlich zu folgender Betrachtung ein:

Look round the world: Contemplate the whole and every part of it: You will find it to be nothing but one great machine, subdivided into an infinite number of lesser machines, which again admit of subdivisions, to a degree beyond what human senses and faculties can trace and explain. All these various machines, and even their most minute parts, are adjusted to each other with an accuracy, which ravishes into admiration all men, who have ever contemplated them. The curious adapting of means to ends, throughout all nature, resembles exactly, though it much exceeds, the productions of human contrivance; of human design, thought, wisdom, and intelligence. Since therefore the effects resemble each other, we are led to infer, by all the rules of analogy, that the causes also resemble; and that the author of nature is somewhat similar to the mind of man. (19)

Cleanthes' methodischer Zugang ist von einer empiristischen Grundhaltung geprägt: "Look round the world «; hier wird allein auf das Beobachtbare gesetzt, und Cleanthes zufolge vermittelt dieses den Eindruck gewollter Ordnung. Doch seine Beschreibung verliert zunehmend »the whole and every part of it " aus den Augen, um an deren Stelle »one great machine" samt untergeordneter, aber ebenso komplexer Aggregate zu setzen. Unter der Hand beginnt nun der Zielpunkt der Analogie die Eingangsbeobachtung zu überlagern, nicht ohne die theologische >Ausstiegsklausel< zu formulieren: dass nämlich die Konstruktion wohl abgestimmter Maschinen dem gut geeichten `Ganzen ‘ zwar ähnele, aber der homo faber im »curious adapting of means to ends « vom " author of nature « bei weitem übertroffen werde. ${ }^{7} \mathrm{Da}$ -

\footnotetext{
${ }^{7}$ In Anlehnung an und zugleich gegen Eberhard Jüngel formuliert: •bei noch so großer Ähnlichkeit zwischen Gott und Welt eine immer noch größere Unähnlichkeit‘; siehe aber Eberhard JÜnGEL, "Meine Theologie - kurz gefaßt, in: DERS., Wertlose Wahrheit. Theologische Erörterungen III, Tübingen ${ }^{2} 2003$ (1990), 1-15, hier: 9, der es genau anders herum ausdrückt.
} 
mit hat Cleanthes die Zutaten beisammen, um die göttliche Variable zu berechnen: Wie ein Uhrmacher einen verlässlichen Chronographen konstruiere und damit als »intelligent cause « (40), d.h. als Träger planvoller Absichten angesehen werden könne, so fungiere auch Gott als causa prima, deren Wirkung von Cleanthes als ebenso geordnet aufgefasst würde wie der Lauf einer Uhr. In einem quasi mathematischen Dreisatz könne nun von den als ähnlich unterstellten Wirkungen und der im einen Fall bekannten Ursache mit großer Wahrscheinlichkeit auf den transzendenten "author" geschlossen werden.

Cleanthes betont eigens, sich ausschließlich der allseits akzeptierten Autorität der »rules of analogy « - in diesem Fall zwischen Design/Designer und Universum/Schöpfer - zu bedienen. Dies mag ihm nicht zuletzt deshalb dringlich erscheinen, weil er mit seinem argument from design ${ }^{8}$ nicht nur im Sinne des Deismus etwas über einen Aristotelischen ersten unbewegten Beweger vorlegt, sondern eine ambitioniertere Richtung einschlägt, wenn er per analogiam auch Gehaltvolles über das Wesen dieses Bewegers zu behaupten versucht (vgl. 47). Für ihn jedenfalls ist es ausgemacht, dass ein Theismus dieses Zuschnitts die alleinige Erklärung des Daseins und Soseins unseres Universums bilde, weshalb er recht optimistisch und zufrieden feststellen kann:

By this argument a posteriori, and by this argument alone, do we prove at once the existence of a deity, and his similarity to human mind and intelligence. (20; vgl. 78.91)

\section{II.2. Philos Einspruch}

Der Skeptiker Philo scheint Cleanthes' zweiter These, wonach »by this argument alone" Gottes Existenz und Wesen bewiesen werden könne, durchaus zuzustimmen; denn dies erlaubt ihm (zumindest Cleanthes gegenüber), die Berechtigung jedes religiösen Glaubens, der seinen Namen verdient, zu untergraben, wenn das argument from design widerlegt ist. Genau dies zu tun, ist Philos Absicht, zumal er die erste These seines Kontrahenten ja gerade bestreitet: Wasserdicht sei dessen Argument keineswegs. ${ }^{9}$

${ }^{8}$ Es ist sicher deutlich, dass die Art des Arguments, wie es von Cleanthes vorgetragen wird, zwar über Familienähnlichkeiten zum kosmologischen sowie teleologischen Gottesbeweis verfügt, sich jedoch keinem der beiden Beweise reibungslos zuordnen lässt. Das argument from design teilt mit ihnen (gegenüber dem ontologischen Argument) die aposteriorische Note und das Interesse an der Ordnung der Welt, wohingegen es sich nicht auf Kausalketten beruft, die - um einen regressus zu umgehen - traditionell zur Annahme einer causa prima führen. Vielmehr wird hier von Ähnlichkeitsbeziehungen ausgegangen, die abduktiv aus dem Wesen des Designers dessen Existenz folgern lassen, nicht umgekehrt.

9 Auch der Name Philos ist eine Anspielung, wohl auf einen der Lehrer Ciceros, zumal Konsens darüber besteht, dass Ciceros De Natura Deorum Hume als Vorbild für seine Dialogues gedient hat. Eine andere Herkunft des Namens vertritt hingegen Rudolf Lüthe, der ihn auf einen 
Hume jedoch hat nun den kritisch-gedankenreichen Erwägungen Philos im ersten Teil der Dialoge ein methodisches Präludium vorgeschaltet. In diesem Vorspiel lässt er Philo wiederholt die Begrenzungen und Schwächen unserer Vernunft hervorheben, um endlich zur Aussichtslosigkeit des Unternehmens einer natürlichen Religion (und Theologie) fortzuschreiten. Dies erläuternd bedient sich auch Philo einer analogen Folgerung: Sei schon der $\mathrm{Zu}$ sammenhang einzelner Teile oft nur schemenhaft erklärbar, erscheine der Versuch, über den Ursprung des Universums Verlässliches in Erfahrung zu bringen, erst recht hoffnungslos (vgl. 7). Mit solchen Manövern der Theologie bewegten wir uns nur jenseits unserer epistemischen Kapazität. Daher dränge sich eine auf den gesamten Lebensvollzug ausgreifende Skepsis als Notwendigkeit auf (vgl. 11).

Cleanthes muss nun widersprechen, will er sein Vorhaben nicht schon aus rein formalen Gründen diskreditiert sehen. Er stellt dabei jedoch Überlegungen an, die von seinen theistischen Absichten unabhängig sind. So verweist Cleanthes mit Recht darauf, dass sich ein generalisierender Zweifel selbst untergrabe und weder wünschenswert sei, noch lebenspraktisch verwirklicht werden könne (vgl. 8f.). Radikale Zweifler glichen daher »a sect of jesters or railers « (13), weil ihre skeptische Devise, an möglichst allem zu zweifeln, der eigenen Praxis, de facto vieles als selbstverständlich anzuerkennen, zuwiderlaufe. Philo wittert jedoch darin den unangebrachten Einspruch eines Konservativen und bedient sich schließlich des Bonmots von Francis Bacon, wonach in Zeiten der Krise Neues lediglich als Abfall vom Alten angesehen werde (vgl. 15). ${ }^{10}$

Dieser Einstieg verschafft den sich dann anschließenden und bereits skizzierten Ausführungen Cleanthes' eine Vorschussplausibilität, gegen die der eigentlich überlegene Philo seinen ganzen Scharfsinn aufbieten muss, um allmählich die Vorherrschaft in diesem Streitgespräch zu gewinnen. In

\footnotetext{
Skeptiker der sneuen Akademie‘, nämlich Philon von Larissa, bezieht; siehe Rudolf LÜTHE, David Hume. Historiker und Philosoph, Freiburg/München 1991, 93.

10 Dass Philo tatsächlich der radikale Skeptiker ist, für den ihn Cleanthes zu halten scheint, und ob letzterer dem Verweis auf eine lebenspraktische Skepsis nicht zustimmen könnte, wäre allerdings zu fragen. Interessant ist jedoch, dass es der Theist ist, der hier eine Position vorbringt, welche Humes eigener überaus nahe kommt. Es sei dafür auf Humes Unterscheidung zwischen akademischer und skeptischer Philosophie verweisen (David Hume, An Enquiry concerning Human Understanding and Other Writings (1748), hg. v. Steven Buck Le, Cambridge 2007, part 12). Hume selbst trat in Anspielung auf die Schule Platons für ein akademisches Denken ein. Diesem erweist der Schotte in mehrfacher Hinsicht Referenz, indem er sich zu religionsphilosophischen Fragen abschließend in Dialogform äußert. Es handelt sich demnach um eine gemäßigte Art skeptischen Denkens, die "Anwalt des praktischen Lebens « sei (Jens KULENKAMPFF, Hume. Einführung, München ${ }^{2} 2003$, 22), insofern "philosophical decisions are nothing but the reflections of common life, methodized and corrected « (Hume, Enquiry, 142); die skeptische Philosophie hingegen ähnelt der hier von Cleanthes abgewiesenen Position eines (Hyper)Cartesianismus; zum philosophiegeschichtlichen Hintergrund siehe Markus GabrieL, Antike und moderne Skepsis, Hamburg 2008, Teil 1, bes. 77-86.
} 
seiner Kritik des argument from design gibt sich Philo als ein skeptischer Denker zu erkennen, welcher der zuvor von Cleanthes geforderten gedanklichen Umsicht und praktischen Verantwortung sehr wohl entspricht, indem er die Dynamik der vorgebrachten analogia auf ihre Grenzwerte hin ausreizt. ${ }^{11}$ Eine Analogie gewinnt an Stärke, je ähnlicher sich die in Beziehung gesetzten Verhältnisse sind, während ihr Erklärungswert korrelativ abnimmt - an diesem Pol der Analogie droht der Anthropomorphismus (vgl. 44.47). Eine Analogie büßt hingegen an Validität ein, je unähnlicher sich das besagte Verhältnis gestaltet, wobei der Erklärungswert wiederum korrelativ gesteigert wird - an diesem Pol der Analogie droht die Unkenntlichkeit Gottes (vgl. 35). Im einen Fall kann Weniges sicher erklärt, im anderen Vieles unsicher dargelegt werden.

Auf diese beiden analogen Extrempunkte lassen sich nun die zahlreichen Einwände, die Philo im Verlauf des Gesprächs vorbringt, verteilen. Ich zähle die wichtigsten Momente auf - zunächst diejenigen, die die Ähnlichkeit der Analoga hervorheben:

- Gesteigerte Ähnlichkeit: Wenn der Uhrmacher und Gott einander ähneln, müsste man mit dem Anthropomorphismus ernst machen und nicht nur von Gottes Auge, sondern auch von seiner Augenbraue sprechen; ${ }^{12}$ zumindest wäre es nötig zu begründen, warum diese Fortsetzungen unerlaubt sind (vgl. 20.42.44.47.101).

- Alternative Erklärungen: Wenn wir schon zum Zweck der Analogie von der Welt ausgehen, bieten sich Erklärungen ihres Soseins an, die dem, was wir aus der Beobachtung kennen, näher liegen und nicht Bezug auf eine nur analog erschlossene Entität nehmen: etwa Zeugung und Wachstum, also selbsterhaltende Prinzipien (vgl. 38.50.52f.59f.), bzw. plurale Erklärungen, die innerhalb der Analogie zum Polytheismus führen würden (vgl. 44.56).

11 Es muss mindestens zwischen zwei Grundformen der Analogie unterschieden werden. Die analogia attributionis drückt die Ähnlichkeit verschiedener Gegenstände oder Personen mit Bezug auf eine oder mehrere Eigenschaften aus. Eine Eigenschaft $x$ wird verschiedenen Dingen $(a, b, c, \ldots)$ zugesprochen, die sich in allen weiteren Eigenschaften unterscheiden können (nicht müssen) $[x$ : $a, b, c, \ldots .$. . Die analogia proportionalitatis hat die Struktur eines Verhältnisses von Verhältnissen. Zwei Dinge $(a, b)$ verhalten sich zueinander wie zwei andere Dinge $(\mathrm{c}, \mathrm{d})$ [a:b $\approx \mathrm{c}: \mathrm{d}]$; dazu: Eberhard Jüngel, Gott als Geheimnis der Welt, Tübingen ${ }^{6} 1999, \mathbb{S} 17$ : »Das Problem analoger Rede von Gott ", 357-383. Bei Cleanthes' Analogie handelt es sich offenbar um die zweite Art, was für die Struktur Philos Kritik von Belang ist. Vgl. grundsätzlich zum Analogiedenken: Karen Gloy, Versuch einer Logik des Analogiedenkens, in: Dies./M. Bachmann (Hg.), Das Analogiedenken. Vorstöße in ein neues Gebiet der Rationalitätstheorie, Freiburg i.Br. 2000, 298-323.

$12 \mathrm{Zu}$ diesem Beispiel siehe auch Ludwig Wittgenstein, Vorlesungen über den religiösen Glauben, in: DERS., Vorlesungen und Gespräche über Ästhetik, Psychoanalyse und religiösen Glauben, hg. v. Cyril Barrett. Deutsch von Ralf Funke, Düsseldorf/Bonn 1994, 77-101, hier: 100. 
- Problem des Bösen: Gehen wir also konsequent von der Beschaffenheit der Welt aus, stellen sich deren Elemente gerade nicht als "adjusted to each other with an accuracy, which ravishes into admiration all men « dar (s.o.). Oft ist das Gegenteil der Fall, sodass Vertreter wie Cleanthes bestenfalls die Vereinbarkeit zwischen dem allmächtigen Gott und dem »mixed bag " (William James) seiner Schöpfung aufzeigen könnten, nicht aber, wie eigentlich nötig, die Ableitbarkeit Gottes aus dieser Welt (vgl. bes. 77.85f.).

Nun zu den Momenten, die auf die Unähnlichkeit der Analoga setzen und - theologisch gewendet - zur apophatischen Tradition gehören:

- Verschiebung des Problems: Die von Cleanthes (und seinen Nachfolgern) angesetzte Ordnung des Universums verschiebe nur das Problem, weil der analog geschlossene Ordner seinerseits nach seinem Ursprung fragen ließe (vgl. 55).

- Überschreitung möglicher Erfahrung: Die wiederum von Cleanthes und Philo geteilte empiristische Grundannahme, nach der Erkenntnis stets als erfahrungsbasiert zu denken sei, stehe mindestens 'windschief`zum Versuch, die Welt samt ihrer Entstehung zu erklären, weil die >Welt ein Grenzbegriff, jedoch kein Gegenstand möglicher Erfahrung bilde (vgl. 18f.45.53f.).

- Fehlende Parallelen: Die von Cleanthes analogisierten Fälle (zwischen Uhren oder Häusern sowie der Welt oder dem Universum) unterscheiden sich im wesentlichen darin, dass wir für erstere ähnlich gelagerte Parallelen kennen, während dies für zweite nicht gilt; doch benötigen wir, so wiederum die empiristische Überzeugung, mehrere gleichartige Instantiierungen eines Falls, um ein induktiv gerechtfertigtes Wissen von ihm erlangen zu können (vgl. 20.26.28).

Nach der anschaulich erläuterten und rhetorisch geschickten Darlegung seiner Reserve ist für Philo die besagte Vorschussplausibilität der Cleanthischen Position ganz aufgebraucht, sodass er den zehnten Teil siegesgewiss beschließt: »Here I triumph « (77).

\section{II.3. Kritik der Kritik - oder: D.Z. Phillips trifft Philo}

Es stellt nun eine gängige Strategie dar, die vielfältigen Erwägungen Philos auf unterschiedliche argumentative Ebenen zu verteilen. ${ }^{13}$ Ein aufschlussreicher Vorschlag dieser Art stammt vom Religionsphilosophen Dewi Z. Phillips (1934-2006), der drei Level der Kritik unterscheidet, die in ihrer Durchschlagskraft zunehmen. Ich werde diese Level vorstellen, um

13 Siehe zum Beispiel John C.A. GASKIn, Hume's Philosophy of Religion, London/Basingstoke 1978, 9-40, der fünf Ebenen differenziert. 
dann auf die für unsere Zwecke wichtige Revision Phillips' in seiner Bewertung der dritten Ebene aufmerksam zu machen. ${ }^{14}$

(i) Zweideutigkeit von Wirkungen und Ursachen: Wenn man gemäß der Analogie davon ausgeht, dass ähnliche Wirkungen ähnliche Ursachen besitzen (vgl. 13.41), sich die Wirkungen jedoch sehr different gestalten, tun es eben auch die Ursachen. Insofern die Welt eine auch von Schlechtigkeit, Gebrechen und Unglück heimgesuchte Welt ist (siehe den gesamten Teil X), kann auch Gott als deren Schöpfer lediglich über einen 'mixed character`verfügen. ${ }^{15}$

(ii) Zirkularität der Design-Analogie: Die Welt als designed aufzufassen, lege das Problem eines designer nahe. Doch bereits dieser erste Zug sei keineswegs alternativlos: Zum einen drängen sich für die komponierte Ordnung andere Erklärungen auf (s.o.) bzw. könnte einen reinen Zufall diesseits nötiger Erklärungen darstellen, zum anderen ginge die zunächst metaphorische Aussage vom Welt-Design in eine ontologische über: Sie lege gerade nahe, was eigentlich in Frage steht: ein designtes System (vgl. z.B. 59-61). ${ }^{16}$

(iii) Kontextlosigkeit der Hypothese: Es stellt sich die Schwierigkeit, eine sinnvolle Analogie zwischen klassifizierbaren Gegenständen, etwa einem Haus, und dem zu keiner Klasse gehörenden - also >klassenlosen $<$ - Universum zu ziehen. Für Häuser haben wir vergleichbare und schon verglichene Erfahrungen von deren Beschaffenheit und Entstehung entlang eines Planes. Für das Universum hingegen lässt sich kein Kontext benennen, in dem solch ein erfahrungskonstituierender Verund Abgleich des Universums als einem großen, noch unfertigen $X$ mit etwas anderem denkbar sei (vgl. 23f. \& bes. 28).

Die Level (i) und (ii) gehören auf diejenige Seite der Analogie, die wie im vorigen Abschnitt gezeigt - in einen kruden Anthropomorphismus

14 Ein guter Überblick zu den drei Level findet sich in: Dewi Z. Phillips, Religion without Explanation, Oxford 1976, 10-12, 22; DERS., Is Hume's True Religion a Religious Belief, in: DERs./Timothy Tessin (Hg.), Religion and Hume's Legacy, London/Basingstoke 1999, 81 98, hier: 83; DERs., Recovering Religious Concepts. Closing Epistemic Divides, Houndmills/ Basingstoke/London 2000, 65.

15 Vgl. Phillips, Religion without Explanation (s.o. Anm. 14), 13, wobei er bereits hier darauf hinweist, dass die erhofften pro/contra-Evidenzen insuffizient bleiben (können), wie John Wisdom mit seiner berühmten Parabel vom unsichtbaren Gärtner erläutert: John W ISD OM, Gods, in: Ders., Philosophy and Psycho-Analysis, Oxford 1969, 149-168, hier: 154-156. PhilLIPS bezieht sich auf die Parabel, ebd. 13-15; ferner DERs., Religion and the Hermeneutics of Contemplation, Cambridge 2001, 59.

16 Vgl. Phillips, Religion without Explanation (s.o. Anm. 14), 14-16, dessen Überlegungen ich hier etwas fortgeführt habe. Bei Phillips selbst heißt es: "Not only is Hume saying that the evidence of design is inconclusive, he is saying that it is misleading to speak of evidence of design in these contexts at all. He is telling us to treat nature naturally. If we do so, we shall find that we arrive at our explanations by relating item to item within nature, without having to appeal to any external intelligence." (ebd. 16; Hervorhebung von mir). 
abzugleiten droht, wobei deren Einordnung durch Phillips unverändert bleibt. ${ }^{17}$ Dies steht im Gegensatz zum Level (iii), zu dem er seine Sicht in einer Weise wandelt, die für unsere Zwecke von Relevanz ist. In einem frühen, 1976 erschienenen Text drückt sich Phillips noch so aus:

We cannot say that the universe has been made by someone because we have no experience of universes which have been made or half-made or of unfinished universes. Hume's point is not a purely agnostic one. He is not simply saying that we can never know whether anyone made the universe. He is questioning the intelligibility of such talk. ${ }^{18}$

Für die Sinnhaftigkeit dieser Redeweise benötigten wir eine »past experience", und weil wir über diese nicht verfügen können, handle es sich nicht um einen kontingent-empirischen Umstand, sondern um eine die »logic of inference « betreffende Auskunft. ${ }^{19}$

Später erfolgt die angesprochene Revision, sodass Phillips die Analogie-Kritik Philos wie folgt bewertet:

$[T]$ he origin of ssomething called the world «, does not, as Hume thinks, remain mysterious. Rather, it has been shown to be unintelligible. Hume does not reach this conclusion himself because he did not appreciate the logical implications of the third level of his own criticisms. ${ }^{20}$

Hier nun sieht Phillips Philo genau dem Agnostizismus verhaftet, von welchem er ihn zuvor gerade freigesprochen hatte. Was Hume seinem Protagonisten in den Mund lege, würde nun hinter dem eigentlich erreichten Niveau der Kritik zurück bleiben, sodass Philo sich damit zufrieden gebe, den Ursprung der Welt - und damit diese selbst - als Mysterium zu betrachten. Doch der Versuch, das argument from design für solch weitreichende Erklärungsansprüche einzuspannen, gestaltet sich nicht zufällig als erfolglos, sondern dieses ganze Unternehmen sei, so Phillips, von einem verwirrten Geist geleitet, sodass bereits die (dann enttäuschten) Hoffnungen von einer tiefgehenden Konfusion infiziert seien. Folgen wir Phillips, habe sich Hume selbst missverstanden, sofern er die Reichweite der von Philo vorgebrachten Einwände nicht in vollem Umfang zur Geltung zu bringen verstand. $^{21}$

17 Ich gehe auf (i) und (ii) nicht näher ein; siehe dazu Peter F. Bloemendand, Grammars of Faith. A Critical Evaluation of D.Z. Phillips's Philosophy of Religion, Leuven/Paris/Dudley, MA 2006, Kap. 5.1.; ferner M. Jamie Ferreira, Phillips: An Inheritance Reconsidered, in: Mark Addis/Robert L. Arrington (Hg.), Wittgenstein and Philosophy of Religion, London 2001, 154-166, bes. 159f.

18 Phillips, Religion without Explanation (s.o. Anm. 14), 18f. (Hervorhebung von mir); vgl. ebd., 22.

19 Beide Zitate: ebd. $17 \& 18$.

20 Phillips, Is Hume's True Religion a Religious Belief (s.o. Anm. 14), 85; vgl. ebd. 84f.; auch DERs., Hermeneutics of Contemplation (s.o. Anm. 15), 70.

21 So sieht es auch Hans Julius Schneider, der diesen Gedanken wie folgt festhält: »Keinen der von ihm fingierten Gesprächspartner lässt Hume aber den Gedanken äußern, aus dem damit angesprochenen Unterschied zwischen Dichtung und Wissenschaft ließe sich folgern, das Projekt einer im Geiste der Wissenschaft verfahrenden natürlichen Religion könnte vielleicht schon als Vorhaben zweifelhaft sein, nicht erst in seiner Durchführung. Auch der Skeptiker Philo, der 
In Summa: Sieht Phillips' frühere Interpretation des Levels (iii) HumePhilos Kritik an der durch Cleanthes vertretenen natürlichen Religion als eine grammatische an, weswegen dessen gesamtes Vorhaben ohne Sinn bleibt, berichtigt Phillips das Resultat seiner Hume-Lektüre später dahingehend, dass nun die "intellectualist distortion of belief in God as Creator of the world «22, wie sie sich im argument from design ausspreche, gar nicht durchschaut sei. Dieses Argument enthalte keinen Fehler, der unter anderen Umständen behebbar wäre, sondern lebt von einer wahrheits- und falschheitsunfähigen Verwirrung. ${ }^{23}$ Phillips selbst vertritt die stärkere erste Lesart und wähnt sich daher zunächst in philosophischer Bruderschaft mit Philo, weswegen später seine Enttäuschung spürbar wird, als er bemerken muss, dass die Verwandtschaft wohl doch nur >zweiten Grades ist. $^{24}$

Was bringt all dies für unser Anliegen, Demea zu rehabilitieren, mit sich? Nun, nachdem Cleanthes' metaphysica als ver(w)irrt abgewiesen sind, hätte Philo nun gute Gründe, jeder Religion abzusagen, wenn die ja auch von Cleanthes geteilte, aber völlig verarmte Bivalenz zwischen natürlicher Religion oder dem Ende des Glaubens eine auch nur annähernd vollständige Alternative bildete. Nun bietet Hume jedoch durchaus andere Zugänge zum religiösen Glauben, nämlich einen gewollt-expliziten durch Philo und einen ungewollt-impliziten durch Demea. Letzterem nähern wir uns, indem wir nach Cleanthes auch von Philo Abschied nehmen.

\section{Philos vera religio}

Phillips' umrissene Selbstkorrektur beruht auf der genaueren Beachtung einer Passage, die alle aufmerksamen Leser des letzten Teils der Dialoge zumindest irritieren wird. Denn der bislang so kritisch eingestellte Philo zeigt sich plötzlich zu einem überraschenden Zugeständnis bereit:

sich gerade offen gezeigt hatte für die antike Vorstellung einer auf die Lebenspraxis gerichteten Philosophie, stellt bei Hume nicht die von Cleanthes vorgeschlagene Richtung in Frage [...].« (Hans J. Schneider, Religion, Berlin/New York 2008, 38).

22 Phillips, Is Hume's True Religion a Religious Belief (s.o. Anm. 14), 89.

${ }^{23}$ Zur (recht positivistischen) Unterscheidung zwischen `Fehler und 'Konfusion siehe vor allem: Wittgenstein and Religion, Houndmills/Basingstoke/London 1993, 108; DERs., On Giving Practice its due - A Reply, in: RelSt 31 (1995), 121-127, hier: 122.

24 Der These, dass Hume in Demea einen Charakter schuf, der reicher war, als Hume selbst erkannte, vermag leider auch D.Z. Phillips kaum etwas abzugewinnen, obwohl die religionsphilosophische Konstellation seines Werkes, einen Weg zwischen theistisch-metaphysischen Ansätzen und moralisierend-reduktiven Zugängen zu finden, derjenigen gleicht, die hier mit Demea aufgebrochen werden soll; zum Verhältnis Demea/Phillips siehe Walter va N Her ck, A Friend of Demea? The Meaning and Importance of Piety, in: Andy F. S Anders (Hg.), D.Z. Phillips' Contemplative Philosophy of Religion. Questions and Responses, Aldershot 2007, 125-138, hier: $125,138$. 
You, in particular, Cleanthes, with whom I live in unreserved intimacy; you are sensible, that, notwithstanding the freedom of my conservation, and my love of singular arguments, no one has a deeper sense of religion impressed on his mind, or pays more profound adoration to the divine being, as he discovers himself to reason, in the inexplicable contrivance and artifice of nature. A purpose, an intention, a design strikes everywhere the most careless, the most stupid thinker; and no man can be so hardened in absurd systems, as at all times to reject it. That nature does nothing in vain. [...] And thus all the sciences almost lead us insensibly to acknowledge a first intelligent author. (89-90; Hervorhebung im Orig.)

Sehr viel mehr als dieser "attenuated deism « 25 schwebt wohl auch Cleanthes kaum vor, der die strue religion< immerhin noch mit einem sunverfälschten` Theismus identifiziert (vgl. 39f.91, bes. 99). Dass Philo diesem Votum nun zuzustimmen scheint, stellt eine der interpretativen Herausforderungen der Dialogues dar.

Gewöhnlicherweise werden für eine entsprechende Erklärung zwei Gründe ins Feld geführt: Entweder verweist man auf den zeitgeschichtlichen Kontext von Humes Schriftentum und die sich daraus ergebenden Hürden der Zensur, mindestens aber auf die Fallstricke der Benachteiligung. Diese hat Hume auch zu spüren bekommen, und er wusste sehr genau, dass ihm seine Einwürfe gegen die herkömmlichen theologischen Lehren von den zeitgenössischen Kirchenvertretern Schottlands mehr als übel genommen werden; die Dialogues sind nicht von ungefähr erst 1779 posthum - also: post Hume - erschienen. Oder aber man nimmt auf den Charakter seiner akademischen Skepsis Bezug, um in Philos Version einer vera religio das Element einer Irenik zu erkennen, welche die Harmonie im Sozialen der Rechthaberei im Theoretischen vorzuziehen lehrt. ${ }^{26}$

Beides muss nicht bestritten werden; dennoch hat Philos 'Konversion eine Pointe, die nicht primär nach einer rückläufigen Entschuldigung verlangt, sondern die argumentativ begründet werden kann. Zunächst nahmen seine gegen Cleanthes' Argument gerichteten Einsprüche die Form interner Kritik an: Er ging darin vom Argument selbst aus und führte es immer wieder zu unangenehmen Folgerungen, die in ihrem kumulativen Effekt das Argument unterminierten. Nun aber wechselt Philo die Strategie, indem er offensichtlich eine Minimalkonzeption religiösen Glaubens akzeptiert und sich auf die analog begründete Annahme eines »first intelligent author « einlässt - doch nur, um die gänzliche Leere dieses nur scheinbaren Entgegenkommens zu verdeutlichen:

So little, replied Philo, do I esteem this suspense of judgement in the present case to be possible, that I am apt to suspect there enters somewhat of a dispute of words into this controversy $[\ldots]$. That the works of nature bear a great analogy to the productions of art is evident;

25 So Van A. Harvey, Is There Anything Religious about Philo's `True Religion`?, in: PhiLLips/Tessin (Hg.), Religion and Hume's Legacy (s.o. Anm.14), 68-80, hier: 69 (im Anschluss an J. Gaskin).

26 Siehe dazu William B. Carnochan, The Comic Plot of Hume's 'Dialogues`, in: MPh 85:4 (1988), 514-522, bes. 515 . 
and according to all the rules of good reasoning, we ought to infer $[\ldots]$, that their causes have a proportional analogy. [...] Here then the existence of a DEITY is plainly ascertained by reason; and if we make it a question, whether, on account of these analogies, we can properly call him a mind or intelligence, notwithstanding the vast difference, which may reasonably be supposed between him and human minds; what is this but a mere verbal controversy? (92; zweite und letzte Hervorhebung von mir)

Die längste zusammenhängende Rede, die Philo in den gesamten Dialogen hält, stellt demnach nicht mehr als eine karikierende Schmalspurversion des Glaubens vor, die von jeglicher Lebenspraxis abgekoppelt und allen inhaltlichen Füllungen beraubt ist. Der so lange und erbittert geführte Disput zwischen Theismus und aufkommendem Atheismus implodiert hier zusehends zu einem Streit rein verbaler Natur. Seine zunächst irritierende Positionierung kostet Philo daher nichts, sondern er kostet sie aus - als einen zweiten `Triumph`, der nach den aus Cleanthes' Sicht gezogenen reductiones nun die Irrelevanz der gesamten Debatte nahelegt. ${ }^{27}$

Tatsächlich verbirgt sich hinter Philos Reduktionismus die Moralisierung der Religion, näherhin die aus Humes Werk The Natural History of Religion von 1757 bekannte Moralkritik des Glaubens. ${ }^{28}$ Demnach stehe >die< Religion überall dort in der Gefahr, ’die< Moral zu schwächen, wo sie mehr zu sein beansprucht als die in nur anderem Vokabular ausgedrückte moralische Forderung. ${ }^{29}$ Wo der religiöse Glaube - Hume meint insbesondere dessen enthusiastische, superstitiöse und schwärmerische Auswüchse (vgl. 97f.) - hingegen über diese Grenze hinausgreife, sei Schlimmstes zu befürchten; denn in diesem Fall sieht Hume einen unerbittlichen und dadurch das soziale Zusammenleben zersetzenden Kampf um das individuelle Seelenheil heraufziehen, der Egoismus, Heuchelei und Betrug in der Gesellschaft sät (vgl. 97). Eine vera religio zeitige solche Resultate keineswegs, wie sich Philo beeilt zu versichern, doch liegt darin lediglich die moralisierte Religion, die den Unterschied zwischen Glauben und Handeln völlig einebnet (vgl. 15 mit Bezug auf John Locke).

27 Siehe zu Philos minimalem Religionskonzept M. Jamie Fer Reir A, Hume’s `Mitigated Scepticisms: Some Implications for Religious Belief, in: Phillips/Tessin (Hg.), Religion and Hume's Legacy (s.o. Anm. 14), 47-67, 53. Einen (letztlich unbefriedigenden, sich auf Schleiermacher berufenden) Versuch, Philos Minimalversion in Schutz zu nehmen, bietet HAR VEY (s.o. Anm. 25), 71, 76-78. - Es ist kaum zu übersehen, dass Philos Sicht ganz der sonstigen Charakterisierung des Unterschieds zwischen Glaube und Unglaube (hier - verkürzt - gleichgesetzt mit Atheismus) entgegen steht; denn der zum Glauben Kommende soll doch eine »neue Kreatur " werden (II Kor 5,17), sodass zwischen ihm und seinem alten Ich (und allen anderen salten Nicht-Glaubenden) »eine enorme Kluft« liegt (so wiederum Wittgenstein, Vorlesungen über den religiösen Glauben (s.o. Anm. 12), 77).

28 Siehe David Hume, The Natural History of Religion, hg. v. A. Wayne Colver, Oxford 1976, bes. section IX \& XIV.

29 In dieser Charakterisierung folge ich FERREIRA, Hume's >Mitigated Scepticism< (s.o. Anm. 27), 61. - Auf die sich hier deutlich abzeichnende Traditionslinie, die vom britischen Deismus über Philos vera religio zur Religionsschrift Immanuel Kants verläuft, kann und muss ich hier nicht eingehen. 
Philos Zugeständnis ist in Wahrheit gar keines, sondern eine argumentative Finte. Schon zuvor und in anderem Zusammenhang hatte Philo den lange nichts ahnenden Demea für seine Zwecke eingebunden (vgl. 87f.), während er nun ein ähnliches Spiel mit Cleanthes treibt, der nicht bemerkt, dass sein Freund ihm eben nicht in einer " unreserved intimacy « begegnet, sondern sich in der Zustimmung zu ihm nur ein noch durchschlagenderer Angriff auf ihn versteckt.

Doch dieses Manöver ist weit problematischer als es zunächst scheint. Die sehr schlichte Version der hiesigen Schwierigkeit bildete die Frage, wie die beiden vorgestellten Kritiken Philos zusammenpassen: Wie also kann man die Berechtigung der Analogie torpedieren und zugleich die Analogie gelten lassen, um sie letztlich herunter zu drosseln auf einen im Ergebnis belanglosen Beweis? Die weniger schlichte Version der Schwierigkeit wird sichtbar, wenn wir auf Phillips' Lesarten des Levels (iii) zurückgreifen, sodass sich folgendes Dilemma auftut: Entweder meint Philo, nicht nur die kontingente Fehlerhaftigkeit, sondern die notwendige Unverstehbarkeit der Analogie zwischen klassifizierbaren Gegenständen und dem außerhalb jeder Klasse befindlichen Universum aufgezeigt zu haben, was zugleich die zugestandene vera religio als "unintelligible « aussortieren würde; oder man nähme diese, wie Phillips es später tut, beim Wort, reduzierte damit aber die Durchschlagskraft der Ebene (iii) auf einen nicht mehr begrifflich-grammatischen, sondern argumentativen Einwand. In diesem Fall kritisierte Philo nur die von Cleanthes gebotene Antwort, in jenem Fall stellte er weit grundsätzlicher die Art der Frage in Abrede.

Setzt man die ambitionierte Lesart von Level (iii) an - und das sollten wir nach Abwägung der vorgestellten Einsprüche tun -, ist es um die vera religio der Moral geschehen; auch sie fällt Philos konzeptuellem Angriff anheim, weswegen er sich das fintenreiche Zugeständnis an Cleanthes gar nicht hätte leisten können. In dem also, was Hume-Philo kritisch zerlegt, erringt er auf ganzer Linie den Sieg. ${ }^{30}$ Doch scheint er eben darin die nicht nur von Phillips beklagte Begriffsarmut zu dokumentieren, da er die Zweiwertigkeit der metaphysica des Theismus einerseits und die Reduktionen des Glaubens auf den von einer minima moralia diktierten Rahmen andererseits für alternativlos hält. Tut man dies, ist man geradezu gezwungen, den Glauben an Gott zu verabschieden. Tut man dies nicht, verabschiedet man sich von Cleanthes und Philo. Doch Humes Figurenkabinett ist ja noch nicht ganz aufgebraucht.

30 So Phillips, Religion without Explanation (s.o. Anm. 14), 25. 


\section{Jenseits von Hume: Demea}

Blicken wir nun nach vorn, indem wir kurz zurück schauen. Die Struktur des bisherigen Gedankengangs wird wohl möglichweise zwei miteinander verknüpfte Fragen hervorrufen:

,War es wirklich nötig, überhaupt auf Cleanthes und Philo einzugehen, um das uns eigentlich leitende Interesse an Demea plausibilisieren zu können?; und:

'Lassen die Positionen von Cleanthes und Philo nicht noch derart großen Spielraum für anders gelagerte Auffassungen, dass wir uns zunächst diesen, nicht jedoch den Andeutungen zu Demea widmen müssten?

Setzen wir zum Zweck des Arguments die drei Charaktere als alle wesentlichen Optionen abdeckende Typisierungen an und verteidigen nun gerade deren Außenseiter, der über eine recht grobe Skizze seiner Position kaum hinaus gelangt, ist es sehr wohl notwendig, ex negativo vorzugehen, d.h. die beiden weitaus prominenteren Stellungnahmen von Cleanthes und Philo einer Kritik zu unterziehen.

Dies führt bereits zur zweiten Frage, zumal die eben angesetzte Voraussetzung, nach der die Typisierung tatsächlich vollständig sei, keineswegs selbstverständlich erscheint. Was uns zu dieser Prämisse berechtigt, ist eine Extrapolation ihres jeweiligen Bildes religiösen Glaubens: Cleanthes wird zwar als Vertreter eines klassischen Theismus vorgestellt, doch die Kritik, die Hume dem Skeptiker Philo in den Mund legt, beschränkt sich nicht auf diesen Theismus allein, sondern betrifft dessen Kernelement, welches dieser mit teilweise ganz anders akzentuierten, dem Theismus gar kritisch gegenüber stehenden Ansätzen gerade teilt: ein personalistisches Gottesbild, welches Gott als in einem ontologisch-räumlichen Sinn transzendent vorstellt. Dann drängen sich auch hier die Fragen auf, mit denen Philo den Optimisten Cleanthes konfrontiert: in welchem Sinn ein solches Bild überhaupt verstehbar sei und wie sich die notwendig daran anschließenden Implikationen, zum Beispiel das Problem des Bösen, umgehen ließen. Von derartigen Einsprüchen können trinitätstheologische Denkmuster wie kreuzestheologische Korrekturen nicht einfach freigesprochen werden; auch ihre Vertreter könnten noch den "friends of Cleanthes " angehören, insofern sich auch in diesem Lager allzu oft die theistische Grundüberzeugung eines metaphysisch aufgeladenen Personalismus durchhält. ${ }^{31}$

31 Die treffende Wendung "friends of Cleanthes « stammt wiederum von PHiLlips, Recovering Religious Concepts (s.o. Anm. 14), Kap. 5. Sie bezieht sich auf neuere theistische Entwürfe, insbesondere auf das Werk von Richard Swinburne und John Hick, welches von Philos Kritik mit betroffen ist; siehe v.a. Richard Swinburne, The Justification of Theism (2002), unter: www.leaderu.com/truth/3truth09.html, bes. 5 (zur Gottesdefinition); ganz ähnlich schon: DERS., The Existence of God, Oxford 1979, bes. 8; siehe auch DERS., Is There a God?, Oxford 21998; zu Swinburnes Theodizee vgl. Der S., The Problem of Evil, in: Stuart C. B R Ow N (Hg.), Reason and Religion, Ithaca/London 1977, 81-102; ferner (und direkt auf Phillips reagierend) John Hick, D.Z. Phillips on God and evil, in: RelSt 43 (2007), 433-441, hier bes. 439f. Zu 
Nimmt man von diesem Abstand, böte sich diesseits der generellen Verneinung religiösen Glaubens die reduktionistische Alternative Philos an. Doch auch sie vermag kaum zu befriedigen. Unabhängig von den internen Problemen seiner Manöver ist es mehr als zweifelhaft, ob die Sprache, die Bilder und die Praxis religiösen Glaubens auch nur annähernd akzeptabel beschrieben sind, wenn sie als ganz auf Moral rückführbar präsentiert werden und somit als Ausdruck einer Haltung, einer Einstellung die problematische Nähe zu einem bloßen Subjektivismus aufweisen, welcher den unbedingten Gegenstandsbezug des Glaubens kaum wird einfangen können. ${ }^{32}$

Wofür Cleanthes und Philo stehen, ist daher umfänglicher als ein so kruder wie begrifflich konfuser Theismus auf der einen Seite und eine unbefriedigend-relevanzlose strue religion auf der anderen. Gegen beide nur angeblichen Grundoptionen in der Theologie und Religionsphilosophie wird man für die vera religio, die ihren Namen wirklich verdient, eine »eigene Provinz« zu reservieren haben, welche die von Cleanthes und Philo über ihre Affirmation und Negation hinweg geteilten Voraussetzungen tat-

einem trinitäts- und kreuzestheologischen Entwurf, der sich als dezidiert nicht-metaphysisch versteht, nach meiner Auffassung aber den theistischen Kern sehr wohl enthält: Eberhard J ÜNGEL, Gottes Sein ist im Werden. Verantwortliche Rede vom Sein Gottes bei Karl Barth. Eine Paraphrase, Tübingen ${ }^{2} 1967$ (1966); Jüngels Ausführungen zur Selbstentsprechung Gottes (ebd. 30, 35f., 76), zur immanenten Trinität (ebd. 62: »innertrinitarischer Rückschluß «) oder zur Präexistenz Christi (ebd. 94) ergeben nur vor diesem personalistischen Ansatz Sinn, obgleich es eine Gegenbewegung bei Jüngel (und Barth) gibt in der Bevorzugung des Begriffs der ,Seinsweise < vor dem der >Person< (ebd. 37, 101). Auch Jürgen Moltmanns `Gekreuzigtem Gott haften noch Theismen an, weil dieser Gott in der Figur der Selbstbeschränkung gedacht wird, Beschränkung der Macht aber immer noch vom alten Hintergrund theistischer omnipotentia, auf die dieser Gott nun freiwillig-mitleidend verzichtet, abhängt. Hier ist die Unverstehbarkeit dessen, worauf angeblich verzichtet wird, nicht erfasst; vgl. Jürgen Moltm anN, Der gekreuzigte Gott. Das Kreuz Christi als Grund und Kritik christlicher Theologie, München 1972, v.a. Kap. VI.

32 Auf religionsphilosophischem Feld ist die Moralisierung des Glaubens einflussreich von Richard Braithwaite vertreten worden, wonach das narrative Netzwerk des Glaubens - seine "stories « - als Verstärker moralischer Maximen wirkten; siehe Richard B. BR Aith W Aite, An Empiricist's View of the Nature of Religious Belief, Cambridge 1955, bes. 25f., 31 u.ö. Der Reduktionismus muss nicht immer die Form der Moralisierung annehmen, wie George LiND BECK verdeutlicht hat: The Nature of Doctrine. Religion and Theology in a Postliberal Age, Philadelphia 1984. Lindbeck unterscheidet bekanntlich drei wesentliche Religionstypen, deren zweite Version er die sexpressiv-ausdrucksorientierte nennt (vgl. ebd. 37f.) und die er der Tra-

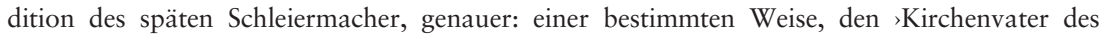
19. Jh.s` zu lesen, zuordnet. Bei allen nötigen Differenzierungen fällt es nicht schwer, in der subjektivitätstheoretisch geprägten Diskussion des deutschsprachigen Bereichs entsprechende Nachfolger zu finden: Wie Braithwaite einem Zwei-Stufen-Denken anhängt, wonach die narrativen Ressourcen des Glaubens sekundäre Ausdrucksformen für eine Weise, das Leben führen zu wollen, sind, so lautet - gemäß Lindbeck (ebd. 22) - die theologische Variante dieser Zweistufigkeit, dass das primäre religiöse Gefühl einer sekundären Symbolisierung zugeführt werde. Verabschiedet man dieses Stufendenken, ergeben sich Formen, die Irreduzibilität religiöser Sprache, Bilder und Praktiken zu denken und damit bei allem notwendigen Reduktionismus einen immer noch ’mehr als notwendigen Nicht-Reduktionismus zu verteidigen. 
sächlich unterläuft und sich somit allein jenseits von Denken und Handeln, jenseits also von Metaphysik und Moral auffinden ließe. ${ }^{33}$ Die Konstellation gleicht folglich derjenigen, die Friedrich Schleiermacher als Ausgangspunkt seiner Reden über die Religion wählte, im Gegensatz zu denen Hume nicht hat erkennen können, dass das durch Cleanthes und Philo personifizierte Entweder/Oder in ein Weder/Noch zu überführen ist. Demea wird uns nun behilflich sein, in eben diese »Provinz« zu gelangen. ${ }^{34}$

\section{IV.1. Demeas Abgang: zwei Erklärungen}

Doch wir hörten bereits, dass es Demea keineswegs in eine neue "Provinz « zu verschlagen scheint; vielmehr sucht er das Weite - »on some pretence or other", wie es am Ende des elften Teils der Dialoge heißt (88). Das eingangs erwähnte sneue Interesse an dem so lange vernachlässigten Demea richtet sich auf eben diesen Vorbehalt, der nun auf unterschiedliche Weisen ausgelegt wird. Obgleich die sneue Hinwendung insofern straditionell bleibt, als sie der Reserve gegenüber Demea letztlich nur neue Munition liefert, kann sie jedoch auch für eine echte Wertschätzung des Außenseiters lehrreich sein. Ich unterscheide dazu zwei Zugänge:

(1) die argumentative Lesart: Dieser Sicht zufolge ist Demea in eine argumentative Enge geführt worden, die ihn gezwungen habe, die Bühne vorzeitig zu verlassen. Hierfür werden wiederum zwei Beobachtungen ins Feld geführt. Zum einen habe insbesondere Philo aufgezeigt, dass alle Gründe, die Demea für den religiösen Glauben als adäquater empfinde als Cleanthes' erfahrungsbasierte Analogie, genauso gut zuungunsten des Glaubens ausgelegt werden könnten; ${ }^{35}$ denn das Böse, das nach Demea zum Glauben führen würde (vgl. 68.75), gefährde nicht nur das argument from design, sondern stelle jeden Zugang zum Glauben vor »Epicurus' old questions «:

Is [God] willing to prevent evil, but not able? then is he impotent [sic.]. Is he able, but not willing? then is he malevolent. Is he both able and willing? Whence then is evil? (74)

Dieser Schwierigkeit könne man zum anderen nicht mit dem Verweis auf Gottes Entzogenheit begegnen. Ganz auf der Linie Cleanthes' Mystik-

$33 \mathrm{Zu}$ den in Cleanthes und Philo personifizierten Positionen und deren über Bejahung und Bestreitung hinweg geteilten Annahmen siehe wiederum Phillips, Recovering Religious Concepts (s.o. Anm. 14), 8; DER s., Religion and Friendly Fire. Examining Assumptions in Contemporary Philosophy of Religion, Aldershot 2004, 50.

34 Friedrich D.E. Schleiermacher, Über die Religion. Reden an die Gebildeten unter ihren Verächtern (1799). Mit einem Nachwort von Carl H. Ratschow, Stuttgart 1997, 26; vgl. ebd. 36.

35 So vor allem James D ye, Demea's Departure, in: HumeSt XVIII:2 (1992), 467-482, hier: 467, 475; zudem sei zu berücksichtigen, dass der von Demea verteidigte sfirst cause noch nicht zum sredeemer eines lebendigen Gottesglaubens führe (vgl. ebd. 477). 
Kritik (s.u.; IV.2.) wird Demea nun vorgehalten, sich in einem Dilemma zu verfangen. Demnach sei es inkonsistent, unsere Vernunft als für die Erfassung Gottes Natur ungenügend anzusehen, zugleich aber seine Existenz als apriorisch darlegbare, d.i. selbstevidente Tatsache anzusetzen. ${ }^{36}$ Wird also von seiner Existenz gesprochen, ist seine Natur ebenso thematisch. ${ }^{37}$

(2) die pädagogische Lesart: Hier nun wird Demeas Abgang vornehmlich als Reaktion der Enttäuschung angesehen. Nicht fehlende Argumente hätten primär zu seinem Verschwinden geführt, sondern die Erkenntnis, von seinen beiden Gesprächspartnern dialogisch hintergangen worden zu sein. Philo und Cleanthes hätten sich während aller Redegänge in einer Allianz befunden, und Philos zwischenzeitliche Nähe zu Demea besonders im zehnten Teil sei nur ein strategisches Arrangement gewesen, um dessen Vorläufigkeit Cleanthes stets gewusst habe. ${ }^{38}$ Der dafür blinde Demea erscheine hingegen als »dogmatic villain of the Dialogues «, ${ }^{39}$ womit zumindest angedeutet ist, wie eine Orthodoxie im Theoretischen zugleich ganz praktisch verblendet. Humes Werk würde gerade darin seine Meisterschaft entfalten, dass es den Leser über die Einsamkeit des Dogmatikers nicht nur in Kenntnis setze, sondern sie bis zu dessen Mitleid für ihn teils ironisch, teils dramatisch inszeniere. ${ }^{40}$

Daraus werden nun Folgerungen gezogen, die vor diesem Hintergrund der intentio auctoris beikommen wollen. Entscheidend sei die dialogisch aufgezeigte Unvereinbarkeit von Demeas Dogmatismus mit einer freien ergebnisoffenen Untersuchung. Diese Inkompatibilität wird entweder aus der Sicht dessen beschrieben, der sich in ihr befindet; ${ }^{41}$ oder sie wird aus der sozialen Perspektive verdeutlicht, sodass die bei Demea vermutete dogmatische Unerbittlichkeit den intoleranten Gegenpol zu einer liberalen Diskurslandschaft bildete, die die »conversation of mankind « (Richard Rorty) gegen jede gesprächslose Erstarrung am Laufen hält. ${ }^{42}$ Demea aber ginge diese Verpflichtung nicht ein, sondern entziehe sich dem Dialog der Reli-

36 Vgl. James Dye, A Word on Behalf of Demea, in: HumeSt XV:1 (1989), 120-140, 121 (wobei James Dye zudem kritisiert, dass sich Demea trotz der behaupteten Selbstevidenz der Existenz Gottes dann doch der Bezugnahme auf die Tradition als einem Autoritätsargument bediene).

37 Siehe Olshewsky (s.o. Anm. 2), 482 f.

38 Siehe bes. Carnochan (s.o. Anm. 26), 519f.

39 Ebd. 516.

40 Es ist das Verdienst dieses (im Detail dann streitbaren) Zugangs, die Hume-Exegese auch auf die wichtigen stilistischen Elemente der Dialoge hin zu öffnen - ein eigentlich ganz naheliegender Zug, schaut man auf die entsprechenden Projekte zum Werk Platos oder Berkeleys. Diese Loslösung der Diskussion von rein argumentativen Erwägungen verdankt sich auch dem neueren literaturwissenschaftlichen Interesse an Hume; dafür stehen u.a. die Beiträge von W.B. Carnochan und D. Rohatyn (s.u. Anm. 42).

41 So beispielsweise bei OLSHEWSKY (s.o. Anm. 2), 490f.

42 Siehe dazu Dennis Rohatyn, Hume's Dialectical Conceits. The Case of Dialogue XII, in: PPR 43:4 (1983), 519-532, bes. 524. 
gionen in fideistischer Manier. ${ }^{43}$ Zum Zwecke eines gedeihlichen Zusammenlebens müsste man, so die erzieherische Pointe, die dialogunfähigen Demeas dieser Welt loswerden.

Was lernen wir aus beiden (sich ja nicht ausschließenden) Lesarten? Dem zweiten Zugang folge ich in dem Versuch, tatsächlich ernst zu nehmen, dass es sich um ein stilistisch eigentümliches Genre handelt, sodass die innere Relation zwischen Gesprächsform und Argumentationsgehalt zu verdeutlichen ist. Dazu möchte ich aber nicht länger eine unterstellte Autorenabsicht eruieren, sondern werde von unseren möglichen Reaktionen auf Humes Dialoge ausgehen - von unseren Anläufen also, dieses uns als Leser einbeziehende Gespräch uns anzueignen. Die erste Lesart hingegen bringt zum Vorschein, gegen welche argumentativen Muster Demea in Schutz zu nehmen ist, soll die Rehabilitierung dieses »dogmatic villain « nicht in einer Schwebe verbleiben, die den Kontakt zum Text längst verloren hat. Es gilt darum, diese Muster anhand der Dialogues wenn auch knapp zu präzisieren.

\section{IV.2. Ein dritter Weg?}

Die Hindernisse, mit denen eine Rehabilitierung Demeas zu kämpfen hat, sind allerdings akut, blickt man auf die Auskünfte, die die Dialoge zu ihm geben, und die Art, wie sie ihn dabei oft nur en passant charakterisieren. So stellen sich formale wie auch positionelle Aspekte gegen eine umstandslose Wertschätzung seiner Auffassung.

In formaler Hinsicht erscheint Demea weit eher als »disciple einer unhinterfragten Sicht auf seinen Glauben, dessen unterlassene Problematisierung ihn notorisch davon abhält, die Dialoge als "philosopher « tatsächlich zu Trialogen werden zu lassen. ${ }^{44} \mathrm{Ihm}$ geht die empiristische Zuversicht Cleanthes', die sich in dessen argumentativen Bemühungen ja deutlich spiegelt, genauso ab wie Philos intellektuelle Beweglichkeit, deren Einfallsreichtum auch vom Gegner honoriert wird (vgl. 7). Demea aber weiß über weite Strecken des Gesprächs kaum etwas beizutragen, manchmal offenbart er gar korrekturbedürftige Unwissenheit (vgl. 69). Von Philo lässt er sich in eine ganz asymmetrische Allianz gegen Cleanthes einspannen, um diesem verdecktem Spiel recht spät und dadurch um so enttäuschter gewahr zu werden (vgl. 87). Es mag schließlich der Eindruck eines Mannes entstehen, der abstrakteren Debatten nicht in gedanklicher Souveränität zu folgen oder sie gar zu gestalten vermag, weil er stets als Anwalt eines unverhandelbaren Dogmatismus auftritt, während doch gerade der radikal Undogmatische den Typus des wirklich Erfahrenen präsentiert. ${ }^{45}$

43 So Prince (s.o. Anm. 3), 305.

44 Vgl. Dye, A Word on Behalf of Demea (s.o. Anm. 36), bes. 127.

45 So sieht es zumindest Hans-Georg Gad A MER, Wahrheit und Methode. Grundzüge einer philosophischen Hermeneutik, Tübingen ${ }^{6} 1990$ (1960), 361. 
Trotz allem hat der in diesem Sinn sunerfahrene` Demea seine Überzeugungen, auf die vor allem Cleanthes sogleich abweisend reagiert: Sofern man die Unerkennbarkeit Gottes derart hervorhebt, wie es Demea tue, beteilige man sich de facto an einer Verneinung Gottes, so Cleanthes zum Auftakt des vierten Teils, weil eine Bejahung seiner Existenz außerhalb jeder verantwortbaren Reichweite liege (vgl. 35):

Or how do you mystics, who maintain the absolute incomprehensibility of the deity, differ from sceptics or atheists, who assert, that the first cause of all is unknown and unintelligible? (ebd.)

Wenig später tauscht Cleanthes diese Frage durch eine bloße Feststellung ein (vgl. 36), welche der Mystik Demeas stets pejorativ begegnet, zumal dessen analogievergessene theologia negativa in eine selbstvergessene negatio Dei übergehe; denn entweder sei Gott für uns verstehbar oder der unverstehbare Gott gleiche einem inexistenten Referenten, so offenbar die von Cleanthes angesetzte Alternative. Sie also bildet den Grund für seine harsche Kritik an dem, was er als mystische Tradition ansetzt.

Demeas Sicht lässt sich nun auf drei Problemfelder hin spezifizieren, die in ihrem Akzent mindestens verdächtig erscheinen werden. Erstens wird er in dezidierter Abgrenzung zu Cleanthes' argument from design, das auf aposteriorische Überlegungen setzt, als Vertreter des ontologischen Arguments vorgestellt - eines Arguments, das offenbar tatsächlich als Beweis veranschlagt wird, womit vorsichtigere Interpretationen unberücksichtigt blieben. Zweitens betreibt anscheinend auch Demea - wie Cleanthes zuvor - das, was ich die Epistemologisierung religiöser Fragen nennen werde; Gott wird demnach zu einem Gegenstand, wobei dieser Gegenstand als unerkennbar beschrieben wird und diese Unerkennbarkeit schließlich als ein erkenntnistheoretisches Problem formuliert ist. Drittens besingt Demea im Duett mit Philo - die gänzliche Negativität der Welt und scheint die lebensweltlichen Phänomene im Blick auf die Religion auf natürliche und moralische Schlechtigkeit hin zu vereinseitigen.

Die genannten formalen Hindernisse werte ich als Humes implizite Positionierung gegenüber der Figur Demeas, näherhin als seine fehlende Sympathie für dessen Form von Rechtgläubigkeit, die ihn davon abhielt, Demea als 'Figur des Dritten< weitere Konturen zu verleihen. Ich werde mich deshalb auf die drei eben skizzierten positionellen Probleme fokussieren, die die im vorangehenden Abschnitt vorgestellte argumentative Lesart, so hoffe ich, konkretisiert haben. Ihnen also soll eine Interpretation ad bonam partem gegeben werden, die vor einer willkürlichen Eisegese verbleibt, indem eine von Hume nicht kontemplierte Möglichkeit Beachtung findet. 


\section{IV.3. Zur Rehabilitierung Demeas}

Ausgangspunkt der Reserve gegenüber Demea ist seine von Cleanthes als widersprüchlich empfundene Mystik gewesen. Zwar kann dieser den drohenden Widerspruch zwischen der behaupteten Unerkennbarkeit Gottes und der dann doch in Anspruch genommenen, wenn auch fragmentarischen Kenntnis seines Wesens herausarbeiten. Auffallend bleibt aber, dass ganz traditionelle Aspekte der mystischen Tradition in den Dialogen gar keine Rolle spielen. Demea wird folglich nicht als jemand vorgestellt, der sich auf gesonderte religiöse Erfahrungen berufen würde, eines inneren privaten Datums also, welches in eine Vereinigung mit Christus bzw. in eine Verschmelzung des Seelenfunkens mit Gott führen würde. Wofür Demea tatsächlich steht, ist die im religiösen Glauben zu überwindende (nicht: aufzulösende) Ferne Gottes, wobei zu klären wäre, von welcher Art diese Distanz ist. Beschreibt man diese als die Unerkennbarkeit religiöser 'Sachverhalte (matter of facts), gerät man zurück in die Untiefen, aus denen wir uns befreien sollten; denn es handelte sich dann um ein Bild des Glaubens, das diesem ein sonst als fahrlässig verabschiedetes Fürwahrhalten ganz ungesicherter Hypothesen unterstellt - etwa Annahmen bezüglich der Entstehung der Welt oder ihrer Fortentwicklung. Nach dieser pseudo-wissenschaftlichen Lesart überstiegen diese Annahmen die Grenzen unserer Sprache und die Kapazitäten von Vernunft und Urteilskraft.

Jedoch könnte sich Demea in diesen Passagen auch für eine ganz anders gelagerte ,Unerkennbarkeit Gottes« stark machen, nach der die besagte Distanz keineswegs unser Erkenntnisvermögen notorisch überfordert; eher bestünde die Schwierigkeit darin, dass die befremdliche Distanz in einer uns abhanden gekommenen Nähe liegt, in einer Intimität also, für die uns oft die Sprache fehlt; Demeas Mystik würde dann nicht auf eine erkenntnistheoretische Kluft aufmerksam machen, sondern auf einen verschütteten Welt- und Selbstbezug, für den wir das Vokabular verloren haben:

Demeas These [lautet] also nicht, dass uns die einschlägigen Dinge so ferne stünden, dass die Worte nicht bis zu ihnen reichten, vielmehr meint er, die für einen smittleren Abstand gemachten Worte für alltägliche Gegenstände seien für etwas, das uns so nahe stehe, unangemessen. ${ }^{46}$

Die SSprache des Glaubens` also brächte uns (wieder) in Kontakt mit den durch sie bedeutungsvollen smatter of facts<, die mitten sin unserem Leben liegen ${ }^{47}$ : Schöpfung, Leid und Freude, Anfang und Ende des Lebens. Deren Geheimnis kommt in dieser sprachlich gestifteten Nähe nun

46 Schneider (s.o. Anm. 21), 40, dem ich hier folge; Schneider ist einer der ganz wenigen, die ein Interesse an Demea haben, ohne ihm eine echte Wertschätzung vorzuenthalten. Einen anderen Versuch, Demea positiv zu lesen, bietet Ira SINGER: http://www.concentric.net/ isinger/ research/demea.html.

47 ,Im Leben liegen $<$ - dies ist eine Wendung von Ludwig Wittgenstein: Zettel, in: Ders., Werkausgabe, Bd. 8, Frankfurt a.M. ${ }^{6} 1994,259-443$, hier: $\$ 533$. 
gerade so zur Sprache, dass es unverfügbar bleibt; denn sich des "great and inexplicable riddle" bemächtigen zu wollen, so Demea, gliche ohnehin einer Gott verneinenden »impiety « $(33 \& 17)$. - Geht man von dieser dem unverfügbar-fernen Gott sehr nahen Mystik aus, verschieben sich zugleich die sich daraus im Detail ergebenden Kritiken am rechtgläubigen Demea:

(1) Zum Plädoyer für einen apriorischen Gottesbeweis: Demea reagiert auf die unüberbrückbaren Schwierigkeiten, aposteriorische Erwägungen zum Beweis Gottes Existenz doch noch zum Erfolg zu führen, indem er grundsätzlich an der Sinnhaftigkeit von induktiven und probabilistischen Ansätzen zweifelt (vgl. 20 \& 63). Eine derart ausgerichtete Handhabung des Problems könne nur einen Gott der Wahrscheinlichkeit hervorbringen, wohingegen Gott im Glauben gewiss ist, in der Anfechtung fremd, im Zweifel fraglich. ${ }^{48}$ Nach diesem begrifflichen Einspruch steuert er auf eine recht konventionelle Skizze des kosmologischen Gottesbeweises zu, fokussiert auf dessen apriorischen Kern, dem notwendig existierenden Anfang aller Kausalketten: ${ }^{49}$

The argument, replied Demea, which I would insist on, is the common one. Whatever exists must have a cause or reason of its existence; it being absolutely impossible for anything to produce itself, or be the cause of its own existence. In mounting up, therefore, from effects to causes, we must either go on in tracing an infinite succession, without any ultimate cause at all, or must at last have recourse to some ultimate cause, that is necessarily existent. (63)

Demea wählt den zweiten Weg und wird dafür ebenso konventionell von Cleanthes kritisiert, indem dieser auf die Unvereinbarkeit des Existenzprädikats mit dessen Notwendigkeit als modalem Status verweist: Wie der aus seinem >dogmatischen Schlummer erweckte Kant bringt schon der hiesige Theist vor, dass die Existenz von $X$ stets die Möglichkeit von non- $X$ einschließt (vgl. 64f.). ${ }^{50}$

Diesen letzten Punkt muss Demea jedenfalls dann nicht bestreiten, wenn Notwendigkeit nicht im rein logischen Sinn verstanden wird, sondern als eine Auskunft, die ihrerseits notwendig wurde, um mit dem nur wahr-

48 Dies scheint Swinburne in seiner Reformulierung des teleologischen Arguments ganz aus den Augen zu verlieren, sodass er gemäß seiner eigenen Standards nur zu einer höheren Wahrscheinlichkeit Gottes Existenz gelangt als zum Gegenteil; siehe Swinburne, The Existence of God (s.o. Anm. 31), 142-150. Dies kann man mit interner Kritik bestreiten (so John L. MACKIE, The Miracle of Theism. Arguments for and against the Existence of God, Oxford 1982, bes. Kap. 8 [c]), doch drängen sich auch externe Einwände auf, die diesen aus induktiven Verfahren gewonnenen Gott der Wahrscheinlichkeit als am Wesen des Glaubens vorbeidenkendes Projekt ablehnen (die durch einige europäischen Großstädte fahrenden Busse mit der Aufschrift »Probably, God does not exist« sind die begrüßenswerte Karikatur dieser Konfusion).

49 Zum Hintergrund des von Demea vorgetragenen Arguments bei Samuel Clarke sowie der Bewertung, inwiefern es tatsächlich 'konventionell ist, siehe: DYE, A Word on Behalf of Demea (s.o. Anm. 36), 122, 127; Ders., Demea's Departure (s.o. Anm. 35), 469; dagegen OlshewSKY (s.o. Anm. 2), 482.

50 Siehe Immanuel Kant, Kritik der reinen Vernunft. Nach der ersten und zweiten Originalausgabe (A: 1781/B: 1787), hg. v. Jens Timmer mann, Hamburg 1998, 670f. (A 594f./B 622f.). 
scheinlichen Gott Schluss zu machen. Gottes Notwendigkeit zu behaupten, hieße dann, einer Glaubensgewissheit Ausdruck zu verleihen, die einer sensiblen Beschreibung der Innenperspektive von Gläubigen fraglos näher kommt als die Implikate der theistischen Beweisführung.

Diese Innenperspektive nimmt Demea als (Recht)Gläubiger selbst ein. Man darf nicht übersehen, dass er sich keineswegs aus einer quasi neutralen Position heraus äußert, sondern >leidenschaftlichく (Kierkegaard) von seinem Glauben spricht. Bereits damit tritt er in Opposition zu Philo und Cleanthes, die methodisch darin überein kommen, dass Glaubensfragen passionslos geführt werden können und dass die in diesen Fragen auf dem Spiel stehende Nähe und Ferne Gottes nun gerade auf Distanz zu halten wäre. Träfe dieses - aus den Naturwissenschaften gewonnene, doch schon für sie fragwürdige - Bild auch nur in Umrissen zu, müssten Gottesbeweise in Stringenz zum Glauben führen. Sie tun es nicht, weil auch Argumente in bestimmter Weise sim Leben liegen - einer Weise, die sich wohl möglich wandelt, wenn man am Glauben als einem entscheidenden, d.i. in die Entscheidung rufenden Moment dieses Lebens teilhat; und so stellt etwa Norman Malcolm, ein Verfechter des ontologischen Arguments, fest:

At a deeper level, I suspect that the argument can be thoroughly understood only by one who has a view of that human 'form of life' that gives rise to the idea of an infinitely great being, who views it from the inside not just from the outside and who has, therefore, at least some inclination to partake in that religious form of life. ${ }^{51}$

Man muss hier nicht sofort das Gespenst eines sich abkapselnden Fideismus wittern, sondern kann in diesem Votum eine hermeneutische Schubumkehr erkennen, sodass auch Demeas Argument nicht kontextlos glaubensstiftend wirkt, wie es vermeintlich wasserdichte Belege zugunsten einer Theorie täten. Der Beweis plausibilisiert also nicht einen argumentativ abgestützten Glauben, sondern umgekehrt bildet der Beweis eine mögliche Explikation des schon Geglaubten; er führt nicht in eine von ihm unberührte Praxis, sondern allein in diesen praktischen Zusammenhängen hat der Beweis seinen >Sitz im Leben ; bei Anselm war es das Gebet, bei Demea ist es >Demut und Frömmigkeit $<$ (vgl. 6f.). ${ }^{52}$

(2) Zur Gefahr der Epistemologisierung: Unter der Epistemologisierung religiöser Fragen verstehe ich die fehlgeleitete Transformation existentiell-lebensweltlicher Probleme in erkenntnistheoretische Herausforderungen. Dabei wird Gottes Unerkennbarkeit von einer grammatischen Aussage über das Konzept ‘Gott $<$ und dessen Eingeflochtenheit in eine verzweigte Glaubens- und Frömmigkeitspraxis in ein Bedauern darüber umgewandelt,

51 Norman Malcolm, Anselm’s Ontological Arguments, in: PhRev 69:1 (1960), 41-62, hier: 62 (Malcolms eigentliches Anliegen, eine Version des ontologischen Arguments, das gegen Kant immun bleibt, vorzustellen, ist hingegen gescheitert, genauso wie seine These verfehlt ist, nach der Anselm zwei (ontologische) Argumente vertreten hätte; vgl. ebd., 41 \& 45).

52 Zur glaubenspraktischen Einbettung von Anselms Argument siehe Ingolf U. D A LFE R TH, Gott. Philosophisch-theologische Denkversuche, Tübingen 1992, 51-94, bes. 59, 62, $66 \mathrm{f}$. 
dass Gott in einem räumlichen Sinne unsere kognitiven Fähigkeiten übersteige. Akzeptieren wir diese Epistemologisierung, die Gott zu einer verobjektivierbaren Entität - zu Deutsch: einem `Dingsda - erklärt, biegen wir sogleich in diejenige Sackgasse ein, an deren Ende die "friends of Cleanthes « warten.

Mit Rudolf Bultmann kann man davor nur warnen: »Kein leichtfertiges Reden von Gottes Unerkennbarkeit! «53 Der Leichtfertigkeit zu entsagen, heißt nun aber nicht, in (mystisches) Schweigen zu verfallen. Worauf es in diesem Zusammenhang ankommt, ist, Demeas Kritik als eine begriffliche wahrzunehmen, sodass deren Status Philos Einwänden gegen Cleanthes gleicht - jedenfalls gemäß deren strengerer Lesart (siehe nochmals die drei Ebenen bei Phillips: Abschnitt II.3.). Eben dieses kritische Niveau ist auch in Demeas nur rudimentären Auskünften angelegt: Demnach begeht Cleanthes - stets in der Gefahr, in den Anthropomorphismus abzugleiten nicht einfach einen vermeidbaren Fehler, sondern der >rechtgläubige< Demea drückt sein Unbehagen an der konfusen Vorgangsweise aus:

I shall be so free, Cleanthes, said Demea, as to tell you, that from the beginning I could not approve of your conclusion concerning the similarity of the deity to men; still less can I approve of the mediums, by which you endeavour to establish it. (20)

Demea stellt hier das ganze >Medium Gottes Unerkennbarkeit verhandelt, um damit eine Sicht auf den Glauben zu öffnen, die die Geheimnisse der Existenz nicht (weg)erklärt, sondern dem bleibenden $»$ wonder at existence ${ }^{54}$ Ausdruck verleiht. Die Undurchdringlichkeit so unzähliger Lebensphänomene - die Probleme des Bösen, die Unverhofftheit des Guten - führt der Glaube demnach keineswegs einer sonst ausstehenden und nun doch noch geglückten Herleitung zu; vielmehr gibt er uns die anderenfalls verlorenen Mittel, die diese Phänomene nicht auf Distanz - dem >mittleren Abstand - halten, sondern uns nahe bringen, gerade indem die Ferne des uns ganz Nahen angenommen wird. Dieser Glaube erklärt nicht das noch Unerklärte, sondern stiftet einen verantwortlichen Umgang mit dem Unerklärbaren; er versichert nicht zuerst die unumstößliche Wabrheit, um dann im Leben orientieren zu können, sondern indem dieser Glaube durchs Leben leitet, ist er für diejenigen, die ihr >Herz an ihn hängen<, wahr. ${ }^{55}$

Demea könnte für diese theologisch und religionsphilosophisch so brisante wie wohl noch ausstehende Wende stehen, die bei allen nötigen argumentativen Bemühungen den Zugang zum Glauben auf die Kontexte hin

53 Rudolf Bultmann, Theologische Enzyklopädie, hg. v. Eberhard Jüngel/Klaus W. MüLLER, Tübingen 1984, 51.

54 Phillips, Is Hume's True Religion a Religious Belief (s.o. Anm. 14), 89.

55 Diese Charakterisierung müsste gegen funktionalistische Missdeutungen in Schutz genommen werden. Dieser Vermutung liegt die Sicht zugrunde, der Glaube wäre eine Option mit prinzipiell gleichwertigen Alternativen. Doch der Glaube ist nicht wählbar-optional, sondern für den Glaubenden eine Gabe. 
öffnet, in denen der Glaube konkret wird: im Gebet, der Andacht, der Ehrfurcht, kurz: der praktisch werdenden Frömmigkeit (vgl. 7.46). ${ }^{56}$

(3) Zur Negativität der Weltbeschreibung: Doch diese Kontexte scheint Demea lediglich in fragwürdiger Auswahl wahrzunehmen und daher das menschliche Dasein ganz einseitig zu beschreiben, blickt man etwa auf Passagen wie die folgenden:

[F]rom a consciousness of his imbecility and misery, rather than from any reasoning, is led to seek protection from that being, on whom he and all nature is dependent. So anxious or so tedious are even the best scenes of life, that futurity is still the object of all our hopes and fears. $[\ldots]$

Weakness, impotence, distress attend each stage of that life: And it is at last finished in agony and horror. $(68-70)$

Wenn Demea (im Verbund mit Philo) den Akzent auf die Schlechtigkeit der Welt legt, tut er dies zunächst in Abgrenzung von Cleanthes' heilem Universum ‘, zu dessen Verteidigung letzterer sich sogar ausdrücklich bereit erklärt, die Faktizität des moralischen Bösen und des natürlichen Übels zu leugnen (vgl. 75f.).

Demea belässt es jedoch nicht bei der Rückgewinnung der Vielfalt lebensweltlicher Phänomene. Er verortet zudem den `Grund der Religion in eben diesen Widerfahrnissen, sodass sowohl Cleanthes' blinder Optimismus, als auch Philos mageres Minimalkonzept 'wahrer Religion`zur Disposition stehen. Demea macht - auf seine Weise überaus tendenziös-negativ - auf die praktischen Hinsichten der Religion aufmerksam. In »devotion «, "worship«", "veneration ", »obedience " (46; vgl. 68) habe der Glaube seinen Ort mitten im Leben und ist darin auf komplexe Weise in ein emotionales Netz aus Hoffnung, Zuversicht, Vertrauen und Ehrfurcht eingebunden (vgl. 70). Das Gefühl der Schwäche, des Elends, der Krankheit stelle den Lebensbezug der Religion - ihre Nähe - her, von deren 'Richtigkeit wir nicht mittels argumentativer Luzidität überzeugt werden, sondern in welche wir in und durch diese Kontexte hineinwachsen (vgl. 68). ${ }^{57}$ So beten $>$ Rechtgläubige nicht, weil sie sich zuvor der wahrhaftigen Existenz des Adressaten versichert hätten, sondern die Wirklichkeit, in der sich der wahrhaft Betende bewegt, ist Gottes unverfügbarer Geist; und preist der >Orthodoxer seinen Schöpfer, hat er sich nicht zuvor die Berechtigung dazu eingeholt, sondern Gott wahrhaft zu preisen, ist die Wirklichkeit, die wir Gott nennen.

Diese drei Momente, die kaum mehr als Andeutungen - aber immerhin An-Deutungen - bilden, werden die eingangs vorgelegte These, so den-

56 Dies scheinen sogar einige seiner Kritiker zuzugestehen; siehe etwa OLSHEWSKY (s.o. Anm. 2), 474, der in Demeas Abgang die Hinwendung zu "concerns with sentiment and character « markiert sieht - was Parallelen zu Humes Moralphilosophie aufweise.

57 In dieser auf das Negative konzentrierten Beschreibung des Ortes der Religion schimmert $\mathrm{Hu}$ mes eigene Sicht durch, die in seinem Werk The Natural History of Religion entfaltet wird und den Schrecken als Grundprinzip der Religion ansieht. 
ke ich, einsichtiger gemacht haben: Demea ist derjenige Charakter aus $\mathrm{Hu}-$ mes Figurenkabinett, der tatsächlich das Treffendste zum religiösen Glauben zu sagen weiß. Der nun ssystematisch Rehabilitierte tritt dabei (auch ohne systematische Habilitation) als derjenige auf, der das religionskritische Erbe Humes zugunsten der Religion authentisch vertritt, indem er gegen alle >Hinterweltler glaubend in dieser Welt verbleibt - einer Welt, die uns nahe kommt, wenn wir ihre Ferne nicht erklärend auf >mittleren Abstand halten.

\section{Gegen Ende: Demeas Rückkehr}

Es fällt nicht immer leicht, zu bemerken, wann es Zeit ist, zu gehen. Wohl möglich flüchtete Demea sogar recht pünktlich - und zwar nicht wegen argumentativer Sackgassen oder dramatisch inszenierter Enttäuschungen, auch nicht um die beruhigende Anonymität gegen das kommunikative Außenseitertum einzutauschen. Doch mag Demea gespürt haben, dass nach der Verhandlung von Cleanthes' Analogie und Philos Minimalkonzept die eigentlichen Quellen eines lebenspraktischen Glaubens, der die doppelt kritische Mitte zwischen metaphysischen Phantasien und moralisierenden Reduktionen hält, noch gar nicht berührt sind - ein Glaube, der durch seinen eigenen, auch eigentümlichen Sprach- und Bildhaushalt ein auf nichts anderes rückführbares Verständnis der Welt und unserer selbst coram Deo zuspielt und beide darin neu qualifiziert.

Die theologische und religionsphilosophische Dringlichkeit, eben diese via media zu finden und auf ihr viel weiter zu gelangen, als ich es hier konnte, wird deutlicher sein, wenn vor Augen steht, wie zahlreich die »friends of Cleanthes « und wie frequentiert ihr in Vielem so verwandter Freundeskreis um Philo eigentlich sind. Beide waren nicht unglücklich darüber, dass Demea »on some pretence or other « abtrat, sodass es kaum überrascht, wenn ihre Nachfolger voraussagen, dass Demea nicht wieder kommen wird. ${ }^{58}$ Als einer seiner Freunde hoffe ich, dass sie im Unrecht bleiben.

\section{ZUSAMMENFASSUNG}

Traditionell wird Demea als das schwächste Glied in Humes berühmten Dialogues concerning Natural Religion angesehen; die Bühne ist ganz dominiert vom optimistischen Theismus, der durch Cleanthes vertreten wird, und den dagegen gerichteten skeptischen Manövern vonseiten Philos. Entgegen diesem traditionellen Bild wird der >orthodoxer Demea nun verteidigt mit der These: Demea hat - von Hume selbst ungewollt und unbemerkt - das Interessanteste zum religiösen Glauben beizutragen; in ihm deutet sich eine Position jenseits der metaphysischen

58 So Carnochan (s.o. Anm. 26), 522; Mackie (s.o. Anm. 48), 226. 
Phantasien des Theismus (und all seiner zahlreichen Nachfolger, den »friends of Cleanthes «) einerseits und Philos Destruktionen, die in eine moralische Minimalversion des Glaubens zu münden scheinen, andererseits an. Es wird deutlich, dass diese Verteidigung keine primär exegetischen Ziele verfolgt, sondern auf eine in Humes Figurenkabinett nur treffend personifizierte Konstellation reagiert, die uns sehen lässt, an welchem Punkt theologisch und religionsphilosophisch auch heute zu arbeiten wäre. Dazu wird nach einer begrifflichen Kritik an Cleanthes und Philo anhand von drei konkreten Beispielen herausgearbeitet, wie ein Bild religiösen Glaubens aussehen könnte, das jenseits der metaphysischen Hoffnungen, die Gott zu einer (Quasi)Person erklären, und der reduktionistischen Zugeständnisse, die in `Gott ‘ lediglich den Ausdruck einer moralischen Einstellung zu erkennen meinen, liegt. Demea steht somit für ein postmetaphysisches Bild religiösen Glaubens - und wir sind eingeladen, zu den »friends of Demea « zu gehören.

\section{SUMMARY}

Traditionally, Demea is considered to be the weakest part in Hume's famous Dialogues concerning Natural Religion; the stage is completely dominated by Cleanthes' optimistic theism and by Philo's sceptical moves already critical of the former. Contrary to this traditional approach, however, the sorthodox Demea will be defended here in maintaining: Demea contributes - neither consciously intended nor recognized by Hume - the most interesting observations concerning religious belief; in him a position is at least alluded lying beyond the metaphysical fantasies of theism (in league with its successors, the »friends of Cleanthes «) on the one hand and Philo's destructions which seem to amount to a moral minimal version on the other hand. It will be clear that this defense is not exegetically orientated; rather, it reacts to a constellation just personalized by Hume's scasting letting us see at which topics we shall continue to work theologically as well as philosophically today. Accordingly and following a conceptual critique of Cleanthes and Philo it will be elaborated by three concrete examples how a picture of religious belief could look like located beyond metaphysical hopes turning God to a (quasi)person and reductivist concessions regarding ‘God as a mere expression of a moral attitude. Demea, however, represents a postmetaphysical picture of religious belief - and we are invited to belong to the "friends of Demea «. 